\title{
Korteweg-deVries equation for longitudinal disturbances in coasting charged-particle beams
}

\author{
Ronald C. Davidson \\ Plasma Physics Laboratory, Princeton University, Princeton, New Jersey 08543, USA
}

(Received 6 January 2004; published 20 May 2004)

\begin{abstract}
This paper employs a one-dimensional kinetic model to investigate the nonlinear longitudinal dynamics of a long coasting beam propagating through a perfectly conducting circular pipe with radius $r_{w}$. The average axial electric field is expressed as $\left\langle E_{z}^{s}\right\rangle=-e_{b} g_{0} \partial \lambda_{b} / \partial z-e_{b} g_{2} r_{w}^{2} \partial^{3} \lambda_{b} / \partial z^{3}$, where $g_{0}$ and $g_{2}$ are constant geometric factors, and $\lambda_{b}(z, t)=\int d p_{z} F_{b}\left(z, p_{z}, t\right)$ is the line density. Assuming a waterbag distribution for the longitudinal distribution function $F_{b}\left(z, p_{z}, t\right)$, it is shown that weakly nonlinear disturbances moving near the sound speed evolve according to the Korteweg-deVries equation.
\end{abstract}

DOI: 10.1103/PhysRevSTAB.7.054402

PACS numbers: 41.75.-i, 05.45.-a, 52.25.Dg

\section{INTRODUCTION}

High energy accelerators and transport systems [1-4] have a wide variety of applications ranging from basic research in high energy and nuclear physics, to applications such as spallation neutron sources, heavy ion fusion, and medical physics, to mention a few examples. It is therefore important to develop an improved basic understanding of the nonlinear dynamics and collective processes in intense charged-particle beam systems. While there have been significant advances in three-dimensional numerical and analytical studies of the nonlinear VlasovMaxwell equations describing intense beam propagation, there is also considerable interest in the development and application of simplified one-dimensional kinetic models to describe the longitudinal dynamics of long coasting beams [5-12]. This paper employs a one-dimensional kinetic model recently developed by Davidson and Startsev [12] for a long coasting beam propagating through a perfectly conducting circular pipe with radius $r_{w}$ (Sec. II). The average axial electric field is expressed as $\left\langle E_{z}^{s}\right\rangle=-e_{b} g_{0} \partial \lambda_{b} / \partial z-e_{b} g_{2} r_{w}^{2} \partial^{3} \lambda_{b} / \partial z^{3}$, where $e_{b}$ is the particle charge, the constants $g_{0}$ and $g_{2}$ are geometric factors that depend on the shape of the transverse density profile and location of the conducting wall, and $\lambda_{b}(z, t)=$ $\int d p_{z} F_{b}\left(z, p_{z}, t\right)$ is the line density. Assuming a waterbag distribution [13-16] for the longitudinal distribution function $F_{b}\left(z, p_{z}, t\right)$, it is shown that weakly nonlinear disturbances moving near the sound speed evolve according to the Korteweg-deVries (KdV) equation (Sec. III). The classical KdV equation [17-21] of course arises in several areas of nonlinear physics (e.g., hydrodynamics, plasma physics, etc.) in which there are cubic dispersive corrections to sound-wave-like signal propagation. The nonlinear $\mathrm{KdV}$ equation also has the appealing feature that it is exactly solvable $[19,21]$ using inverse scattering techniques. Earlier treatments by Karpman et al. [22] and Bisognano [23] have also developed theoretical models leading to the Korteweg-deVries equation for weakly nonlinear disturbances in neutral plasma-loaded waveguides [22] and for intense beam propagation in a circular pipe [23]. An important difference between the present paper and these treatments [22,23] is that the present analysis provides a rigorous derivation of the $\mathrm{KdV}$ equation that makes use of the self-consistent kinetic model for longitudinal beam dynamics recently developed by Davidson and Startsev [12] that incorporates the important effects of transverse density profile shape, longitudinal beam thermal effects, etc. As such, the present analysis should have a wide range of applicability for weakly nonlinear disturbances moving slightly above the sound speed in intense charged-particle beams.

This paper considers longitudinal disturbances in a long coasting beam with characteristic radius $r_{b}$. The beam is made up of particles with charge $e_{b}$ and rest mass $m_{b}$ propagating in linear geometry (the $z$ direction) with directed axial kinetic energy $\left(\gamma_{b}-1\right) m_{b} c^{2}$, where $\gamma_{b}=\left(1-\beta_{b}^{2}\right)^{-1 / 2}$ is the relativistic mass factor, $V_{b}=$ $\beta_{b} c$ is the average axial velocity of the beam particles, and $c$ is the speed of light in vacuo. It is assumed that the beam propagates through a straight, perfectly conducting cylindrical pipe with wall radius $r_{w}$, and the applied transverse focusing force $\mathbf{F}_{\text {foc }}^{\mathrm{tr}}$ is modeled in the smoothfocusing approximation. Finally, the nonlinear dynamics of the beam particles is treated in the thin-beam (paraxial) approximation, and the particle motions in the beam frame are assumed to be nonrelativistic $[1,12]$.

\section{THEORETICAL MODEL AND ASSUMPTIONS}

In the present analysis, we adopt a one-dimensional kinetic model recently developed by Davidson and Startsev [12] that describes the self-consistent nonlinear evolution of the longitudinal distribution function $F_{b}\left(z, p_{z}, t\right)$, the average self-generated axial electric field $\left\langle E_{z}\right\rangle(z, t)$, and the line density $\lambda_{b}(z, t)=\int d p_{z} F_{b}\left(z, p_{z}, t\right)$. For simplicity, the analysis is carried out in the beam frame (unprimed variables), and the beam intensity is assumed to be sufficiently low that the beam edge radius $r_{b}$ and rms radius $R_{b}=\left\langle r^{2}\right\rangle^{1 / 2}$ exhibit a negligibly small dependence on line density $\lambda_{b}$. In addition, beam properties such as the number density $n_{b}(r, z, t)$ of beam 
particles are assumed to be azimuthally symmetric about the cylinder axis $(\partial / \partial \theta=0)$, where $(r, \theta, z)$ are cylindrical polar coordinates with $x=r \cos \theta, y=r \sin \theta$, and $r=\left(x^{2}+y^{2}\right)^{1 / 2}$. Finally, the axial spatial variation in the number density $n_{b}(r, z, t)$ and line density $\lambda_{b}(z, t)=$ $2 \pi \int_{0}^{r_{w}} d r r n_{b}(r, z, t)$ is assumed to be sufficiently slow that $k_{z}^{2} r_{w}^{2} \ll 1$, where $\partial / \partial z \sim k_{z} \sim L_{z}^{-1}$ is the inverse length scale of the $z$ variation.

Within the context of these assumptions, the onedimensional kinetic equation describing the nonlinear evolution of the longitudinal distribution function $F_{b}\left(z, p_{z}, t\right)$ and the average axial electric field $\left\langle E_{z}^{s}\right\rangle(z, t)$ can be expressed correct to order $k_{z}^{2} r_{w}^{2}$ in the beam frame as [12]

$$
\begin{gathered}
\frac{\partial}{\partial t} F_{b}+v_{z} \frac{\partial}{\partial z} F_{b}+e_{b}\left\langle E_{z}^{s}\right\rangle \frac{\partial}{\partial p_{z}} F_{b}=0, \\
e_{b}\left\langle E_{z}^{s}\right\rangle=-e_{b}^{2} g_{0} \frac{\partial \lambda_{b}}{\partial z}-e_{b}^{2} g_{2} r_{w}^{2} \frac{\partial^{3} \lambda_{b}}{\partial z^{3}},
\end{gathered}
$$

where the geometric factors $g_{0}$ and $g_{2}$ are defined by

$$
\begin{gathered}
g_{0}=2 \int_{0}^{r_{w}} \frac{d r}{r}\left(2 \pi \int_{0}^{r} d r r \frac{n_{b}}{\lambda_{b}}\right)^{2}, \\
g_{2}=\frac{2}{r_{w}^{2}} \int_{0}^{r_{w}} \frac{d r}{r}\left(2 \pi \int_{0}^{r} d r r \frac{n_{b}}{\lambda_{b}}\right) \int_{0}^{r} d r r \int_{r}^{r_{w}} \frac{d r}{r} \\
\times\left(2 \pi \int_{0}^{r} d r r \frac{n_{b}}{\lambda_{b}}\right) .
\end{gathered}
$$

Here, we have assumed a perfectly conducting cylindrical wall with $\left[E_{z}^{s}\right]_{r=r_{w}}=0$ and consider the class of axisymmetric, bell-shaped density profiles $n_{b}(r, z, t)$ of the form

$$
n_{b}= \begin{cases}\frac{\lambda_{b}}{\pi r_{b}^{2}} f\left(\frac{r}{r_{b}}\right), & 0 \leq r<r_{b}, \\ 0, & r_{b}<r \leq r_{w} .\end{cases}
$$

In Eqs. (1)-(5), $\lambda_{b}=\int d p_{z} F_{b}=2 \pi \int_{0}^{r_{w}} d r r_{b}$ is the line density, $r_{b}$ is the edge radius of the beam (assumed independent of $\left.\lambda_{b}\right)$, and $f\left(r / r_{b}\right)$ is the profile shape function with normalization $\int_{0}^{1} d X X f(X)=1 / 2$. As a simple example, for $f\left(r / r_{b}\right)=(n+1)\left(1-r^{2} / r_{b}^{2}\right)^{n}, n=$ $0,1,2, \ldots$, over the interval $0 \leq r<r_{b}$, it can be shown that [12]

$$
\begin{array}{r}
g_{0}=\ln \left(\frac{r_{w}^{2}}{r_{b}^{2}}\right)+\sum_{m=1}^{n+1} \frac{(n+1)}{m(m+n+1)}, \\
g_{2}=\frac{1}{2}\left[1-\frac{1}{n+2} \frac{r_{b}^{2}}{r_{w}^{2}}\left(1+\ell n \frac{r_{w}^{2}}{r_{b}^{2}}\right)\right. \\
\left.\quad-\sum_{m=1}^{n+1} \frac{1}{m(m+n+2)} \frac{r_{b}^{2}}{r_{w}^{2}}\right] .
\end{array}
$$

In Eqs. (5)-(7), $n=0$ corresponds to a step-function density profile; $n=1$ corresponds to a parabolic density profile; and $n \geq 2$ corresponds to an even more sharply peaked profile with $\left[n_{b}\right]_{r=r_{b}}=0=\left[\partial n_{b} / \partial r\right]_{r=r_{b}}$. Note from Eqs. (6) and (7) that the precise values of $g_{0}$ and $g_{2}$ exhibit a sensitive dependence on profile shape [12]. Moreover, the mean-square beam radius is $R_{b}^{2}=$ $\lambda_{b}^{-1} 2 \pi \int_{0}^{r_{w}} d r r r^{2} n_{b}=(n+2)^{-1} r_{b}^{2}$ for the choice of shape function $f\left(r / r_{b}\right)=(n+1)\left(1-r^{2} / r_{b}^{2}\right)^{n}, n=0,1,2, \ldots$.

In any case, Eqs. (1) and (2) constitute the starting point in the present kinetic model of longitudinal nonlinear beam dynamics. The detailed wave excitation properties of Eqs. (1) and (2) of course depend on the form of the distribution function $F_{b}\left(z, p_{z}, t\right)$. However, as a general remark, for small-amplitude perturbations (linearization approximation) Eqs. (1) and (2) support soundwave-like disturbances (with signal speed depending on $g_{0}$ and the momentum spread of $F_{b}$ ) with cubic dispersive modifications (depending on $g_{2}$ ) [12]. For present purposes, we specialize to the class of exact nonlinear solutions to Eq. (1) corresponding to the waterbag distribution [13-16]

$$
F_{b}\left(z, p_{z}, t\right)= \begin{cases}A=\mathrm{const}, & -m_{b} V_{b}^{-}(z, t)<p_{z}<m_{b} V_{b}^{+}(z, t), \\ 0, & \text { otherwise }\end{cases}
$$

for $-\infty<z<\infty$ (infinitely long coasting beam). Here, the distribution function $F_{b}=A$ remains constant within the interval indicated in Eq. (8) and zero outside, whereas the boundary curves $V_{b}^{-}(z, t)$ and $V_{b}^{+}(z, t)$, assumed single valued, distort nonlinearly as the system evolves according to Eqs. (1) and (2).

It is convenient to introduce the macroscopic fluid quantities corresponding to line density $\lambda_{b}=\int d p_{z} F_{b}$, average axial velocity $V_{b}=\lambda_{b}^{-1} \int d p_{z} v_{z} F_{b}$, longitudinal particle pressure $P_{b}=m_{b} \int d p_{z}\left(v_{z}-V_{b}\right)^{2} F_{b}$, and heat flow $Q_{b}=m_{b} \int d p_{z}\left(v_{z}-V_{b}\right)^{3} F_{b}$, where $v_{z}=p_{z} / m_{b}$ is the axial particle velocity. Some straightforward algebra that makes use of Eqs. (1), (2), and (8) gives the closed system of nonlinear fluid equations for $\lambda_{b}(z, t), V_{b}(z, t)$, and $P_{b}(z, t)$ corresponding to

$$
\begin{gathered}
\frac{\partial}{\partial t} \lambda_{b}+\frac{\partial}{\partial t}\left(\lambda_{b} V_{b}\right)=0, \\
\lambda_{b}\left(\frac{\partial V_{b}}{\partial t}+V_{b} \frac{\partial}{\partial z} V_{b}\right)+\frac{1}{m_{b}} \frac{\partial P_{b}}{\partial z}=-\frac{\lambda_{b} e_{b}^{2} g_{0} \frac{\partial \lambda_{b}}{m_{b}}}{\partial z} \\
-\frac{\lambda_{b} e_{b}^{2} g_{2} r_{w}^{2} \frac{\partial^{3} \lambda_{b}}{m_{b}},}{\partial z^{3}} \\
\left(\frac{\partial}{\partial t}+V_{b} \frac{\partial}{\partial z}\right)\left(\frac{P_{b}}{\lambda_{b}^{3}}\right)=0 .
\end{gathered}
$$

Here, for the choice of waterbag distribution in Eq. (8), $\lambda_{b}=A m_{b}\left(V_{b}^{+}-V_{b}^{-}\right), \lambda_{b} V_{b}=(1 / 2)\left(V_{b}^{+2}-V_{b}^{-2}\right), P_{b}=$ $(1 / 12) m_{b} A\left(V_{b}^{+}-V_{b}^{-}\right)^{3}$, and $Q_{b}=0$ (exactly), which provides closure of the fluid equations. We therefore express 
[see Eq. (11)]

$$
P_{b}(z, t)=\frac{P_{b 0}}{\lambda_{b 0}^{3}} \lambda_{b}^{3}(z, t),
$$

where $P_{b 0}=$ const and $\lambda_{b 0}=$ const represent the unperturbed pressure and line density, respectively, and $P_{b 0} / \lambda_{b 0}^{3}=1 / 12\left(m_{b} A\right)^{2}=$ const. Here, $A$ is the constant phase-space density in Eq. (8).

\section{DERIVATION OF KORTEWEG-DEVRIES EQUATION}

In the subsequent analysis we introduce the constant speeds $U_{b T}, U_{b 0}$, and $U_{b 2}$ defined by

$$
U_{b T}^{2}=\frac{3 P_{b 0}}{\lambda_{b 0} m_{b}}, \quad U_{b 0}^{2}=\frac{\lambda_{b 0} e_{b}^{2} g_{0}}{m_{b}}, \quad U_{b 2}^{2}=\frac{\lambda_{b 0} e_{b}^{2} g_{2}}{m_{b}},
$$

and the normalized (dimensionless) fluid quantities $\eta(z, t)$ and $U(z, t)$ defined by

$$
\eta=\frac{\lambda_{b}-\lambda_{b 0}}{\lambda_{b 0}}, \quad U=\frac{V_{b}}{\left(U_{b 0}^{2}+U_{b T}^{2}\right)^{1 / 2}} .
$$

In Eqs. (13) and (14), $U_{b T}$ is the thermal speed, $U_{b 0}$ is the effective sound speed associated with the geometric factor $g_{0}$, and $U_{b 2}$ is an effective speed that measures the strength of the cubic dispersive term in Eq. (10) associated with the geometric factor $g_{2}$. Finally, it is convenient to introduce the scaled (dimensionless) time variables $T$ and spatial variable $Z$ defined by

$$
T=\left(\frac{U_{b T}^{2}+U_{b 0}^{2}}{U_{b 2}^{2}}\right) \frac{U_{b 2} t}{r_{w}}, \quad Z=\left(\frac{U_{b T}^{2}+U_{b 0}^{2}}{U_{b 2}^{2}}\right)^{1 / 2} \frac{z}{r_{w}} .
$$

Making use of Eqs. (12)-(15), the nonlinear fluid description provided by Eqs. (9)-(11) reduces exactly to

$$
\begin{gathered}
\frac{\partial}{\partial T} \eta+\frac{\partial}{\partial Z}(U+\eta U)=0 \\
\frac{\partial}{\partial T} U+\frac{\partial}{\partial Z}\left(\eta+\frac{1}{2} U^{2}+\frac{1}{2} \frac{U_{b T}^{2}}{U_{b 0}^{2}+U_{b T}^{2}} \eta^{2}+\frac{\partial^{2}}{\partial Z^{2}} \eta\right)=0 .
\end{gathered}
$$

The fluid description provided by Eqs. (16) and (17) is exactly equivalent to the nonlinear kinetic description provided by Eqs. (1) and (2) for the choice of waterbag distribution in Eq. (8).

The fluid equations (16) and (17) in scaled variables are particularly amenable to direct analysis. For example, for traveling pulse (soliton) solutions we look for solutions to Eqs. (16) and (17) that depend on $Z$ and $T$ exclusively through the variable $Z^{\prime}=Z-M T$, where $M=$ const is the normalized pulse speed measured in units of the sound speed $\left(U_{b 0}^{2}+U_{b T}^{2}\right)^{1 / 2}$. Making use of $\partial / \partial T=$ $-M \partial / \partial Z^{\prime}$ and $\partial / \partial Z=\partial / \partial Z^{\prime}$, Eq. (16) can be integrated once to give $U\left(Z^{\prime}\right)\left[1+\eta\left(Z^{\prime}\right)\right]=M \eta\left(Z^{\prime}\right)$, where use has been made of $U\left(Z^{\prime}= \pm \infty\right)=0=\eta\left(Z^{\prime}= \pm \infty\right)$. Integrating Eq. (17) and substituting $U=M \eta /(1+\eta)$ then gives for $\eta\left(Z^{\prime}\right)$

$$
\frac{\partial^{2} \eta}{\partial Z^{\prime 2}}+\left[1-\frac{M^{2}}{1+\eta}+\frac{1}{2} M^{2} \frac{\eta}{(1+\eta)^{2}}+\frac{1}{2} \frac{U_{b T}^{2}}{U_{b 0}^{2}+U_{b T}^{2}} \eta\right] \eta=0
$$

where $\left[\eta^{\prime \prime}\right]_{Z^{\prime}= \pm \infty}=0$ and $[\eta]_{Z^{\prime}= \pm \infty}=0$ are assumed. For present purposes, we solve Eq. (18) in the weakly nonlinear limit, treating $|\eta| \ll 1$ and retaining terms to order $\eta^{2}$. Introducing the scaled amplitude

$$
\tilde{\eta}\left(Z^{\prime}\right)=\left(\frac{3}{2} M^{2}+\frac{1}{2} \frac{U_{b T}^{2}}{U_{b 0}^{2}+U_{b T}^{2}}\right) \eta\left(Z^{\prime}\right),
$$

Eq. (18) can be approximated by

$$
\frac{\partial^{2} \tilde{\eta}}{\partial Z^{\prime 2}}-\left(M^{2}-1\right) \tilde{\eta}+\tilde{\eta}^{2}=0 .
$$

For $M^{2}>1$ the exact soliton solution to Eq. (20) is

$$
\tilde{\eta}=\frac{3}{2}\left(M^{2}-1\right) \operatorname{sech}^{2}\left[\frac{1}{2}\left(M^{2}-1\right)^{1 / 2}(Z-M T)\right] .
$$

Consistent with the assumption of weak nonlinearity (small amplitude), in Eq. (21) it is assumed that $M-1=$ $\epsilon$, where $0<\epsilon \ll 1$, which corresponds to a compressional pulse $(\tilde{\eta}>0)$ moving slightly above the sound speed $\left(U_{b 0}^{2}+U_{b T}^{2}\right)^{1 / 2}$. In this case, the amplitude of the soliton in Eq. (21) is proportional to $\epsilon$, whereas the soliton width is proportional to $\epsilon^{-1 / 2}$.

The functional form of Eq. (21) strongly suggests that a weakly nonlinear analysis of Eqs. (16) and (17) for general disturbances moving near the sound speed $(M=1)$ may satisfy a Korteweg-deVries-like equation [17-21]. Indeed, for $M-1=\epsilon$, the argument in Eq. (21) can be expressed as

$$
\frac{1}{2}\left(M^{2}-1\right)^{1 / 2}(Z-M T)=\frac{1}{\sqrt{2}}\left[\epsilon^{1 / 2}(Z-T)-\epsilon^{3 / 2} T\right] .
$$

Therefore, for disturbances moving near the sound speed, we introduce the new independent stretched variables $\xi$ and $\tau$ defined by

$$
\xi=\epsilon^{1 / 2}(Z-T), \quad \tau=\epsilon^{3 / 2} T,
$$

and expand Eqs. (16) and (17) according to

$$
\begin{aligned}
& \eta=\epsilon \eta^{(1)}+\epsilon^{2} \eta^{(2)}+\cdots, \\
& U=\epsilon U^{(1)}+\epsilon^{2} U^{(2)}+\cdots,
\end{aligned}
$$

where $\eta^{(1)}, \eta^{(2)}$, etc., are of order unity. Equation (23) then gives $\partial / \partial Z=\epsilon^{1 / 2} \partial / \partial \xi$ and $\partial / \partial T=\epsilon^{1 / 2}(\epsilon \partial / \partial \tau-$ $\partial / \partial \xi)$. Substituting into Eqs. (16) and (17), we obtain 


$$
\begin{aligned}
& \left(\epsilon \frac{\partial}{\partial \tau}-\frac{\partial}{\partial \xi}\right)\left(\eta^{(1)}+\epsilon \eta^{(2)}+\cdots\right)+ \\
& \frac{\partial}{\partial \xi}\left(U^{(1)}+\epsilon U^{(2)}+\epsilon \eta^{(1)} U^{(1)}+\cdots\right)=0,
\end{aligned}
$$

and

$$
\begin{gathered}
\left(\epsilon \frac{\partial}{\partial \tau}-\frac{\partial}{\partial \xi}\right)\left(U^{(1)}+\epsilon U^{(2)}+\cdots\right)+ \\
\frac{\partial}{\partial \xi}\left[\eta^{(1)}+\epsilon \eta^{(2)}+\cdots+\epsilon \frac{\partial^{2}}{\partial \xi^{2}} \eta^{(1)}+\cdots+\right. \\
\left.\frac{1}{2} \epsilon U^{(1)^{2}}+\frac{1}{2} \frac{U_{b T}^{2}}{U_{b 0}^{2}+U_{b T}^{2}} \epsilon \eta^{(1)^{2}}+\cdots\right]=0
\end{gathered}
$$

In Eqs. (25) and (26), we have scaled out a factor $\epsilon^{3 / 2}$ common to all of the terms.

To solve Eqs. (25) and (26), we equate the coefficients of like powers of $\epsilon$ equal to zero. In lowest order, Eqs. (25) and (26) give $\partial \eta^{(1)} / \partial \xi=\partial U^{(1)} / \partial \xi=0$, which can be integrated to give

$$
\eta^{(1)}(\xi, \tau)=U^{(1)}(\xi, \tau)
$$

for isolated disturbances with $\left[\eta^{(1)}\right]_{\xi= \pm \infty}=0=$ $\left[U^{(1)}\right]_{\xi= \pm \infty}$. In order $\epsilon$, Eqs. (25) and (26) reduce to

$$
\begin{gathered}
\frac{\partial}{\partial \tau} \eta^{(1)}-\frac{\partial}{\partial \xi} \eta^{(2)}+\frac{\partial}{\partial \xi}\left(U^{(2)}+\eta^{(1)} U^{(1)}\right)=0, \\
\frac{\partial}{\partial \tau} U^{(1)}-\frac{\partial}{\partial \xi} U^{(2)}+\frac{\partial}{\partial \xi}\left[\eta^{(2)}+\frac{\partial^{2}}{\partial \xi^{2}} \eta^{(1)}+\frac{1}{2} U^{(1)^{2}}+\right. \\
\left.\frac{1}{2} \frac{U_{b T}^{2}}{U_{b 0}^{2}+U_{b T}^{2}} \eta^{(1)^{2}}\right]=0 .
\end{gathered}
$$

Eliminating $(\partial / \partial \xi)\left[U^{(2)}-\eta^{(2)}\right]$ in Eq. (29) by means of Eq. (28), and substituting $\eta^{(1)}=U^{(1)}$, it is readily shown from Eq. (29) that $\eta^{(1)}(\xi, \tau)$ evolves according to

$$
\frac{\partial}{\partial \tau} \eta^{(1)}+\left[\frac{3}{2}+\frac{1}{2} \frac{U_{b T}^{2}}{U_{b 0}^{2}+U_{b T}^{2}}\right] \eta^{(1)} \frac{\partial}{\partial \xi} \eta^{(1)}+\frac{1}{2} \frac{\partial^{3}}{\partial \xi^{3}} \eta^{(1)}=0 .
$$

Finally, introducing $\tilde{\boldsymbol{\eta}}(\xi, \tau) \equiv\left[3 / 2+U_{b T}^{2} / 2\left(U_{b 0}^{2}+\right.\right.$ $\left.\left.U_{b T}^{2}\right)\right] \eta^{(1)}(\xi, \tau)$, Eq. (30) reduces directly to the familiar form of the $\mathrm{KdV}$ equation [17-21] given by

$$
\frac{\partial}{\partial \tau} \tilde{\eta}+\tilde{\eta} \frac{\partial}{\partial \xi} \tilde{\eta}+\frac{1}{2} \frac{\partial^{3}}{\partial \xi^{3}} \tilde{\eta}=0
$$

Equation (31) is the main result of this paper. To summarize, for a coasting beam described by the longitudinal kinetic equations (1) and (2), the present analysis shows for the choice of waterbag distribution in Eq. (8) that disturbances moving near the sound speed $\left(U_{b 0}^{2}+\right.$ $\left.U_{b T}^{2}\right)^{1 / 2}$ satisfy the Korteweg-deVries equation (31). The cubic dispersive term in Eq. (31) can of course be traced back to the term proportional to $\partial^{3} \lambda_{b} / \partial z^{3}$ in Eq. (2). Furthermore, Eq. (31) can be solved exactly using inverse scattering techniques [19,21]. As a general remark, for specified initial disturbance $\eta(\xi, 0)$, a discrete number of soliton solutions emerge from the disturbance moving to the right as $\tau \rightarrow \infty$. These solitons have the form given in Eq. (21) with the tallest (fastest) solitons on the right. That portion of the initial disturbance that does not decompose naturally into solitons, disperses and decays to negligible amplitude as $\tau \rightarrow \infty$.

\section{CONCLUSIONS}

Several points are noteworthy regarding the derivation of the Korteweg-deVries equation in Eq. (31). First, the specific form of $\left\langle E_{z}^{s}\right\rangle$ in Eq. (2), which assumes a perfectly conducting wall at $r=r_{w}$ [12], has played an important role. The inclusion of finite wall impedance $\tilde{Z}$ and/or the effects of a slip factor $\eta$ for a circular (large-aspect-ratio) ring will lead to different equations $[10,11]$ governing the weakly nonlinear evolution of the system. Second, the present nonlinear analysis has been carried out for the choice of waterbag distribution in Eq. (8). If instead the analysis is carried out for perturbations about a Maxwellian distribution $F_{b}^{0}\left(p_{z}\right)$, it is anticipated that there will be an additional term in Eq. (31) describing the weak Landau damping of the disturbance due to resonant wave-particle interactions [20]. This is analogous to the corrections to the Korteweg-deVries equation obtained for weakly damped ion acoustic waves in plasmas [20]. Finally, for simplicity, the present analysis has been carried out in the beam frame. The corresponding results in the laboratory frame can be obtained by Lorentz transformation to a frame of reference moving with axial velocity $-V_{b}=-\beta_{b} c$ relative to the beam frame [12]. Apart from the coordinate transformation, the main modification in the laboratory frame is the replacement of the geometric factors by $g_{0} \rightarrow g_{0} / \gamma_{b}^{2}$ and $g_{2} \rightarrow g_{2} / \gamma_{b}^{4}$, where $\gamma_{b}=\left(1-\beta_{b}^{2}\right)^{-1 / 2}[12]$.

\section{ACKNOWLEDGMENTS}

This research was supported by the U.S. Department of Energy.

[1] R. C. Davidson and H. Qin, Physics of Intense Charged Particle Beams in High Energy Accelerators (World Scientific, Singapore, 2001), and references therein.

[2] M. Reiser, Theory and Design of Charged Particle Beams (Wiley, New York, 1994).

[3] A.W. Chao, Physics of Collective Beam Instabilities in High Energy Accelerators (Wiley, New York, 1993).

[4] See, for example, Proceedings of the 2001 Particle Accelerator Conference (IEEE Catalog No. OICH37268, 2001), pp. 1-4098. 
[5] I. Hofmann, Z. Naturforsch. A 37, 939 (1982).

[6] O. Boine-Frankenheim, I. Hofmann, and G. Rumulo, Phys. Rev. Lett. 82, 3256 (1999).

[7] I. Hofmann, Laser Part. Beams 3, 1 (1985).

[8] L. K. Spentzouris, J.-F. Ostiguy, and P. L. Colestock, Phys. Rev. Lett. 76, 620 (1996).

[9] R. Fedele, G. Miele, L. Palumbo, and V. G. Vaccaro, Phys. Lett. A 179, 407 (1993).

[10] H. Schamel, Phys. Rev. Lett. 79, 2811 (1997).

[11] H. Schamel and R. Fedele, Phys. Plasmas 7, 3421 (2000).

[12] R. C. Davidson and E. A. Startsev, Phys. Rev. ST Accel. Beams 7, 024401 (2004).

[13] R. C. Davidson, H. Qin, S. I. Tzenov, and E. A. Startsev, Phys. Rev. ST Accel. Beams 5, 084402 (2002).

[14] R. C. Davidson, Methods in Nonlinear Plasma Theory (Academic Press, New York, 1972).

[15] F. Hohl and M. R. Feix, Astrophys. J. 147, 1164 (1967).
[16] K.V. Roberts and H. L. Berk, Phys. Rev. Lett. 19, 297 (1967).

[17] D. J. Korteweg and G. deVries, Philos. Mag. 39, 422 (1895).

[18] H. Washimi and T. Taniuti, Phys. Rev. Lett. 17, 966 (1966).

[19] C. S. Gardner, J. M. Greene, M. D. Kruskal, and R. M. Miura, Phys. Rev. Lett. 19, 1095 (1967).

[20] E. Ott and R. N. Sudan, Phys. Fluids 12, 2388 (1969).

[21] See, for example, Methods in Nonlinear Plasma Theory (Ref. [14]), pp. 19-31.

[22] V. I. Karpman, J. P. Lynov, P. Michelsen, H. L. Pecseli, J. Juul Rasmussen, and V. A. Turikov, Phys. Fluids 23, 1782 (1980).

[23] J. J. Bisognano, in Proceedings of the 1996 European Particle Accelerator Conference, Sitges (IOP, Bristol, U.K., 1996), pp. 328-330 